\title{
28 Research Square \\ Quantitative Analysis of Macroscopic Solute Transport in the Murine Brain
}

\section{Lori A Ray}

Montana State University https://orcid.org/0000-0001-9083-7493

\section{Martin Pike}

Oregon Health \& Science University

\section{Matthew Simon}

Denali Therapeutics Inc

\section{Jeffrey J Iliff}

University of Washington School of Medicine

Jeffrey J Heys ( $\square$ jeffrey.heys@montana.edu )

Montana State University

\section{Research}

Keywords: biotransport, brain transport, glymphatic, perivascular transport, interstitial transport, dynamic contrast-enhanced MRI

Posted Date: August 19th, 2021

DOl: https://doi.org/10.21203/rs.3.rs-806107/v1

License: (c) (i) This work is licensed under a Creative Commons Attribution 4.0 International License.

Read Full License

Version of Record: A version of this preprint was published at Fluids and Barriers of the CNS on December 1st, 2021. See the published version at https://doi.org/10.1186/s12987-021-00290-z. 


\section{QUANTITATIVE ANALYSIS OF MACROSCOPIC SOLUTE TRANSPORT IN THE MURINE BRAIN}

Authors: Lori A Ray ${ }^{1}$, Martin Pike ${ }^{2}$, Matthew Simon ${ }^{3,4, \#}$, Jeffrey J Iliff ${ }^{5,6,7}$, Jeffrey J Heys ${ }^{1}$ Affiliations: ${ }^{1}$ Department of Chemical and Biological Engineering, Montana State University; ${ }^{2}$ Advanced Imaging Research Center, Oregon Health and Sciences University; ${ }^{3}$ Department of Anesthesiology and Perioperative Medicine, Oregon Health and Science University;

${ }^{4}$ Neuroscience Graduate Program, Oregon Health and Science University; ${ }^{5}$ VISN 20 Mental Illness Research, Education and Clinical Center (MIRECC), VA Puget Sound Health Care System; ${ }^{6}$ Department of Psychiatry and Behavioral Sciences, University of Washington School

of Medicine; ${ }^{7}$ Department of Neurology, University of Washington School of Medicine; ${ }^{\#}$ Present Address: Denali Therapeutics 


\begin{abstract}
Background: Understanding molecular transport in the brain is critical to care and prevention of neurological disease and injury. A key question is whether transport occurs primarily by diffusion, or also by convection or dispersion. Dynamic contrast-enhanced (DCE) MRI offers a whole-brain view of transport and the potential for quantitative analysis to determine fundamental transport parameters. However, few DCE-MRI studies have utilized this potential, instead reporting parameters with arbitrary units disconnected from fundamental transport processes.
\end{abstract}

Methods: In this work, DCE-MRI experimental data is combined with subject-specific finiteelement models to quantify transport parameters in different anatomical regions across the whole mouse brain. Effective diffusivity $\left(D_{e f f}\right)$, a transport parameter combining all mechanisms of transport, is determined for each region by minimizing the root mean square error between simulations and data. The resulting $D_{e f f}$ sets are compared to apparent diffusivity $\left(D_{a p p}\right)$ to draw conclusions about dominant transport mechanisms in each region.

Results: In the perivascular regions of major arteries, $D_{\text {eff }}$ was over 10,000 times greater than $D_{a p p}$. In the brain tissue, constituting interstitial space and the perivascular space of smaller blood vessels, $D_{\text {eff }}$ was $10-25$ times greater than $D_{a p p}$.

Conclusions: The analysis concludes that convection is present throughout the brain. Convection is dominant in the perivascular space of major surface and branching arteries $(\mathrm{Pe}>$ $10,000)$ and significant to large molecules $(>1 \mathrm{kDa})$ in the combined interstitial space and perivascular space of smaller arteries (not resolved by DCE-MRI). Importantly, this work supports periarterial convection along penetrating and smaller arteries. 
Key Words: biotransport, brain transport, glymphatic, perivascular transport, interstitial transport, dynamic contrast-enhanced MRI

\section{Background}

Interstitial molecular transport is an essential element in physiological brain function, contributing to neurotransmission, ion homeostasis, nutrient delivery and waste clearance (1). Changes in interstitial molecular transport have been implicated in several pathological states, including the proposal that impairment of interstitial peptide and protein clearance may underlie the vulnerability of the aging or injured brain to the development of protein aggregates in neurodegenerative disease (2-4).

While interstitial solute transport is classically attributed to diffusion (5), Rennels et al. (6) and Cserr et al. (7) reported that tracers injected both into the brain interstitium and the cerebrospinal fluid compartments move preferentially along perivascular spaces (PVSs) surrounding the cerebral vasculature and that the observed tracer movement was too fast to be explained by diffusion alone. These PVSs are annular spaces bounded by the vascular wall on the inside and by perivascular astroglial endfeet on the outside. Patterns of tracer distribution demonstrate that PVSs are continuous both with the brain interstitium and the cerebrospinal fluid (CSF) surrounding the brain.

This topic has risen in visibility over recent years with the description of the 'glymphatic' system (3, 8-10). The glymphatic system is a brain-wide system for molecular transport (Figure 1) where: 1) subarachnoid CSF enters the brain along PVS surrounding penetrating arteries (moving in the same direction as blood flow), 2) fluid and solutes exchange into the interstitial space through gaps between astroglial endfeet, and 3) interstitial fluid and solutes drain along 
PVSs surrounding large-caliber draining veins towards sinus-associated CSF compartments. The subsequent characterization of meningeal lymphatic vessels $(11,12)$ associated with dural sinuses provided a final potential step in the clearance of these interstitial solutes from the cranium along the lymphatic drainage. In the glymphatic model, PVSs surrounding the branching cerebral vasculature provide a low-resistance conduit for efficient exchange of fluid between the brain surface and regions deep within the brain, supporting solute delivery and waste clearance.

The existence of both perivascular and interstitial convection in the brain has undergone much debate (14-22). Periarterial flow has been measured directly at $1.2 \mathrm{~mm} / \mathrm{min}(18,19)$ and entrains to arterial pulsation $(9,18,23)$, suggesting that peristaltic flow within the PVS is generated by pulsation of the arterial (inner) wall against a mostly rigid outer 'wall' comprised of ensheathing leptomeninges or astroglial endfeet. However, to date mathematical models of periarterial flow do not replicate the flows observed experimentally $(20,24,25)$, rendering unclear the mechanism of peristaltic PVS flow under the unique properties of the arterial pulse wave. Dispersion, which occurs when flows with no net direction (such as eddies) facilitate mixing and accelerate transport relative to diffusion, has also been predicted to moderately enhance periarterial transport over diffusion alone $(20,26)$. Measurements of periarterial flow have been conducted only along distil segments of the middle cerebral artery (MCA) on the brain surface $(18,19)$. Observed flow characteristics are indicative of flow in an open channel $(18$, 23), which has a lower hydraulic resistance than a porous, media-filled space. However, the 1$\mu \mathrm{m}$ diameter microspheres tracked in the experiments did not enter PVSs surrounding penetrating arteries $(19,23)$. It is possible that periarterial flow may only occur around surface arteries (19) or that periarterial space surrounding penetrating vessels may be "somewhat 
porous", excluding the relatively large microspheres while permitting the transport of large soluble tracers $(8,19,23)$.

In the interstitial space, transport models have estimated interstitial flow superficial velocity ranging from no flow to $0.05 \mathrm{~mm} / \mathrm{min}(27-29)$. An interstitial flow of $0.01 \mathrm{~mm} / \mathrm{min}$ is sufficient to affect the transport rate of macromolecules greater than $1 \mathrm{kDa}$ in size, including the peptides and proteins (amyloid $\beta$, tau, a synuclein) implicated in neurodegenerative diseases (27, 29). Differences between models of interstitial flow originate from varying assumptions about the hydraulic conductivity of brain tissue (the ease with which fluid can move through 'porous' interstitial space) and pressure gradients between periarterial and perivenous spaces, which are not known. With these uncertainties surrounding the existence, magnitude and extent of perivascular and interstitial flow, it is unclear whether convection is a significant contributor to the transport of molecules within brain tissue.

Dynamic contrast-enhanced magnetic resonance imaging (DCE-MRI) has emerged as the gold-standard for investigating molecular transport within the healthy and diseased rodent and human brain, with a recent review summarizing fifteen such studies since the first experiment in $2013(30,31)$. DCE-MRI offers a brain-wide, macroscopic view of transport, complimentary to the microscopic view of flow surrounding single vessels provided by 2-photon microscopy ( 8 , 18). Studies utilizing DCE-MRI demonstrate that contrast agents injected into the subarachnoid CSF move along preferential pathways following major arteries near the surface of the brain, then penetrate into the brain at a rate too rapid to be described by diffusion alone (31-37) (Figure 2). Typically, cluster analysis is performed on time-series signal data to show the speed of contrast agent uptake in different brain regions or the general direction of contrast agent movement $(17,31,38)$. Optimal mass transport (OMT) modelling has emerged as an excellent 
tool for visualizing movement of intrathecal contrast agents through the brain (39). However, these semi-quantitative techniques do not permit the determination of the fundamental parameters characterizing molecular transport that are independent of experimental procedures and allow direct comparison with known diffusion rates. Within these DCE-MRI studies, measured signal changes have been regarded as a surrogate for contrast concentration, assuming a direct proportionality between concentration and signal change, when this relationship is somewhat more complex (see Methods).

In the present study, we develop a computational tool to reduce a complex DCE-MRI data set to a few fundamental values characterizing transport in the brain, which permit comparison to known diffusion rates. Most previous computational models of brain transport predicting fundamental parameters have been at the microscale and have only qualitatively compared results to experimental data $(20,27,28)$. An exception is Valnes et al., who developed a finiteelement model simulating contrast transport in a section of the human brain (40), utilizing DCEMRI data derived from human studies $(41,42)$ to estimate transport parameters in grey and white matter. However, the complexity of the highly-folded surface of the human brain, variability among study participants, and the noisy nature of DCE-MRI data resulted in dependence of results upon boundary-data filtering methods. Here, a finite-element transport model is developed for the simpler case of the mouse brain. The mouse brain has a smoother cortical surface, little white matter, and smaller size that enables modelling of the whole brain while small-animal MRI has the resolution needed to identify the major cerebral vasculature. This permits the quantification of transport along major periarterial conduits, which are important to molecular movement across the brain. Contrast-agent concentrations calculated from DCE-MRI data and simulations from subject-specific, finite-element transport models of the whole brain 
are used to quantify transport parameters in different anatomical regions, describing macroscopic transport mechanisms across the brain volume. This approach provides insight into the properties of glymphatic transport, including whether convective fluid flow is an important contributor to molecular transport within brain tissue.

\section{Methods}

An overview of the process used to estimate transport parameters for different anatomical regions using finite-element modelling and DCE-MRI data is presented in Figure 3. At a high level, DCE-MRI signal data are used to calculate contrast agent concentration and to define discrete anatomical regions for the computational model relevant to brain-wide transport. Subject-specific, finite-element models of molecular transport are built for the whole mouse brain where unique transport parameters are applied to each defined anatomical region. Transport parameter sets are varied and simulations performed to determine the optimal combination of parameters for each subject that minimizes the difference between the concentration data and the simulation. Calculated transport parameters are then compared to the known diffusivity of the contrast agent through brain tissue to draw conclusions about convective transport in the different regions of the brain.

\section{DCE-MRI Experiments}

The present study reflects an analysis of previously published DCE-MRI data collected in n=4 male and female 3-6 month old C57 B1/6 mice (17). Briefly, ketamine/xylazine-anesthetized $(100 / 10 \mathrm{mg} / \mathrm{kg}$, respectively) animals were injected with $10 \mu \mathrm{l}$ of $68 \mathrm{mM}$ gadoteridol contrast agent at a rate of $0.5 \mu 1 / \mathrm{min}$ via a pulled glass pipette surgically inserted through the atlanto- 
occipital membrane, into the cisterna magna. Following contrast agent infusion, a $2 \mu 1$ chase of saline was infused. Serial 3D FLASH T1-weighted MR images with isotropic (100x100x100 $\mu \mathrm{m}$ ) voxels were obtained at $11.75 \mathrm{~T}$ at 10 -minute intervals over $80 \mathrm{~min}$. The image series for each subject was aligned to a baseline image using a linear rigid-body registration, followed by masking to remove non-brain regions, and subsequent linear registration to the Badhwar mouse hippocampal Atlas with 60x60x60 $\mu \mathrm{m}$ isotropic voxels (FSL). As reported in our prior study, early contrast enhancement occurred along the ventral surface of the brain, after which parenchymal enhancement throughout brain tissue began to increase.

Calculation of Contrast Concentration and Identification of Anatomical Regions.

To quantify transport parameters, concentration--a fundamental physical variable--is required. The majority of DCE-MRI data in the literature are reported and analyzed as signal. Measured signal changes are often regarded as a surrogate for contrast concentration, assuming direct proportionality. T1-weighted signal is increased by the presence of the contrast agent, but the relationship between signal change and concentration is more complex. Gadoteridol concentration is related to MRI signal by (43):

$$
[\text { gadoteridol }] \cong \frac{1}{r_{1} \cdot T_{1,0}}\left(\frac{S_{G d^{-}} S_{0}}{S_{0}}\right)
$$

where:

$r_{1}=$ Gadoteridol (Prohance) relaxivity, $3.2 \times 10^{-3} \mathrm{~L} / \mathrm{mmol}-\mathrm{ms}(44)$

$T_{1,0}=$ pre-contrast relaxation time $(\mathrm{ms})$

$S_{G d}=$ signal intensity after contrast injection (as a function of time, dimensionless)

$S_{0}=$ baseline signal intensity prior to contrast agent injection (dimensionless) 
The proportionality assumption between contrast concentration and signal change is correct if $T_{1,0}$ is constant across the sample. However, $T_{1,0}$ is dependent on the molecular environment and varies significantly $(500-4,500)$ across different tissues in a biological subject, especially one as complex as the brain. $T_{1,0}$ can be estimated from baseline MRI signals collected at different flip angles according to the following equation (45):

$$
S\left(M_{0}, \alpha\right)=M_{0} \sin (\alpha) \frac{1-e^{-T R / T_{1,0}}}{1-\cos (\alpha) e^{-T R / T_{1,0}}}
$$

where:

$T R=$ repetition time $(16 \mathrm{~ms}$ for the experiments reported here $)$, and $\alpha=$ flip angle

For each voxel, $T_{1,0}$ is calculated from $S_{0}$ (baseline) images at $\alpha=3^{\circ}$ and $15^{\circ}$ using a curve fit to Equation 2. Knowing $T_{1,0}$, Equation 1 is used to calculate gadoteridol concentration from DCE-MRI signal for each voxel and time point. The calculated concentration, [gadoteridol], is a superficial concentration $(\bar{c})$ used in porous media theory, where $\bar{c}=c \cdot \phi$ and $\phi$ is void fraction or porosity. It is assumed the void fraction is constant across the brain; $\phi$ has a well-known value of approximately $20 \%(1,46)$ for most adult brain tissues. Throughout the text the accent on superficial concentration has been dropped and it is denoted simply as $c$.

Upon careful examination of the concentration data, regions of distinctly different concentration dynamics are observed. These regions correlate with anatomical features of the brain and are observed in all experimental subjects. Concentration and $T_{1,0}$ thresholds were used to develop masks, voxels assigned to a specific feature, that segment the brain into volumes exhibiting different concentration dynamics. Since $T_{1,0}$ varies markedly between different types 
of tissue, it can be used to identify anatomical features, such as blood vessels and ventricles. Using $T_{1,0}$, the segmented volumes (described in Results) are shown to correlate with anatomical features.

\section{Transport Model.}

Transport of molecules in brain tissue may occur by a combination of mechanisms according to Equation 3, including diffusion, dispersion, convection, and source or sink terms (given in order on the right-hand side of Equation 3):

$$
\frac{\partial c}{\partial t}=D_{a p p} \nabla^{2} c+D_{d i s p} \nabla^{2} c-v \cdot \nabla c+s(c)-f(c)
$$

where: $c=$ concentration, $D_{a p p}=$ apparent diffusivity, $D_{\text {disp }}=$ dispersion coefficient, $v=$ superficial velocity (a vector field), $s(c)=$ spatially dependent source term (e.g., injection), and $f(c)=\operatorname{sink}$ term (e.g., efflux route)

Measurements of fluid velocity or pressure gradients throughout the brain and the fine anatomical details $(<60 \mu \mathrm{m})$ needed to solve Equation 3 are not available. However, using T1 mapping, DCE-MRI permits contrast agent concentration within each voxel to be defined as a function of time. Therefore, a simple transport model is utilized:

$$
\frac{\partial c}{\partial t}=D_{e f f} \nabla c^{2}+s(c)
$$

where all transport mechanisms are 'lumped' into a single parameter, effective diffusivity $\left(D_{e f f}\right)$. Effective diffusivity, $D_{e f f}$, represents a composite of diffusion, perivascular dispersion, perivascular convection, and interstitial convection within a single transport parameter. Modelling transport with $D_{e f f}$ under conditions where the dominant mechanisms are uncertain is a general approach that has been taken previously $(40,47)$ to quantify and compare transport 
rates within the CNS. The simplified transport model is also agnostic towards the mechanism of transport, which is advantageous given the broad range of proposed hypotheses, models and results surrounding the subject of brain transport, spanning from models focusing on diffusion, to dispersion, to convection (25). $D_{e f f}$ is expected to be different for each segmented anatomical volume.

The $D_{\text {eff }}$ values are compared to the known apparent diffusivity $\left(D_{a p p}\right)$ of the MRI contrast agent gadoteridol through brain tissue:

$$
D_{\text {app }}=D / \lambda^{2}=0.016 / 1.73^{2}=0.005 \mathrm{~mm}^{2} / \mathrm{min}
$$

where $D=0.016 \mathrm{~mm}^{2} / \mathrm{min}(48)$ is the free diffusivity, or the diffusivity in an open volume, of gadoteridol, and $\lambda=1.6$ (46) or $\lambda=1.85$ (29) is tortuosity, which represents the degree to which molecular transport is slowed by the porous medium. From this comparison, we can infer the prevalence and magnitude of convection and dispersion for each anatomical subdomain in the transport model. For example, if significant convection is present, the $D_{\text {eff }}$ values will be orders of magnitude greater than the $D_{a p p}$ values.

\section{Finite-element Model.}

Three-dimensional, finite-element models (FEMs) of transport in the mouse brain were developed based on the diffusion equation (Eqn. 4). Subject-specific models of the whole brain (skull and subarachnoid space excluded) segmented into five anatomical volumes, or subdomains, were built for four different mice. Effective diffusivities were varied for each anatomical subdomain to determine the optimal combination of parameters that best fit the DCEMRI concentration data. 
To build the finite-element mesh, brain surfaces were extracted from the MRI data using MRIcroS (49). Defects in the resulting surface were repaired using Meshlab (50). The volume mesh was generated with gmsh (51) using tetrahedral elements. The anatomical masks were interpolated onto the mesh using an inverse-distance weighted (IDW) method to define anatomical subdomains within the whole-brain mesh. Time-series contrast concentrations were also interpolated onto the mesh for error calculations. The IDW method is essentially the application of a Gaussian filter, and therefore also had the effect of locally smoothing the data.

The simple model of the diffusion equation (Eqn. 4) was solved by finite-element method within the complex geometry of the whole mouse brain. Effective diffusivity, $D_{e f f}$, was assumed to be constant within each subdomain, but each subdomain may have different values. The contrast injection, which occurred over the first 20 minutes of the experiment, was modelled as a point source in space and a rectangular function in time. Due to enhanced $\mathrm{T} 2 / \mathrm{T} 2 *$ relaxation compromising data on the surface of the brain near the injection site, the concentration at the boundary was not complete and could not be used as the boundary condition. Therefore, a noflux boundary condition was applied. Although some contrast agent will leave the brain via CSF and glymphatic efflux routes, it is assumed this loss will be small over the short timescale of the DCE-MRI experiment.

The appropriateness of the no-flux boundary condition was tested by calculating the total amount of gadoteridol in the brain versus time using the concentrations calculated from DCEMRI data. If a no-flux boundary condition accurately represents the physical situation, the total amount of $\mathrm{Gad}$ in the brain would increase during the injection, over the first 20 minutes, and then remain constant. The amount of gadoteridol in the brain increases or plateaus over the full 
course of the experiment (up to 84 minutes) for three out of the four mice, consistent with no loss of contrast agent through the boundaries, or no flux.

Effective diffusivities were varied among the subdomains and the root mean square error ( $r m s$ ) between the data and the simulation mapped to determine the set of $D_{\text {eff }}$ resulting in the minimum error.

$$
r m s=\sqrt{\sum_{c=1}^{T} \frac{\left(c_{\text {data }}-c_{\text {simulation }}\right)^{2}}{T}}
$$

The $\mathrm{T} 2 / \mathrm{T} 2 *$ interference region, the ventricles, and the blood vessels were excluded from the error calculation. Time points up to 52 minutes were used in the error calculation, later time points were excluded to minimize the error due to assumptions of no efflux routes and the noflux boundary condition.

Equation 4 was approximately solved using FEniCS $(52,53)$, an open-source solver of partial differential equations by the finite-element method, using quadratic (Lagrangian) mesh elements and a preconditioned iterative solver for the linear system of equations. The time derivative was discretized using a backward difference (i.e., an implicit Euler method). The solution for each set of effective diffusivities required 2-3 hours on an Amazon Web Server (AWS) c5 instance or a MacBook Pro with an i9 processor and 32 GB of RAM. Fifty to twohundred combinations of $D_{\text {eff }}$ were simulated for each subject to generate error surfaces from which the values resulting in the minimum rms were determined (see Supplemental Material). Post processing of simulations and DCE-MRI concentration data was carried out using Python (54), Paraview (55), and Excel. Every effort was made to use open-source software.

\section{Results}

Segmentation of Transport Regions 
Careful evaluation of three-dimensional time-series DCE-MRI experimental data revealed several regions of enhancement with distinct concentration dynamics that aligned with the anatomy of the cerebral arterial vasculature and the cerebral ventricular system. Based on these observations, the pre-contrast baseline images and DCE-MRI data were used to individually segment each brain into five volumes: 1) the cerebral ventricles, 2) the macroscopic cerebral arterial vasculature, 3) periarterial spaces surrounding the major surface arteries, 4) periarterial spaces surrounding major branching and penetrating arteries, and 5) the remainder of the brain parenchyma (Figure 4A). These segmentations were used to create subdomains within the finiteelement model for which unique transport parameters were determined.

The MRI acquisition obtained isotropic $100 \mu \mathrm{m}$ voxels, which through atlas registration were interpolated to $60 \mu \mathrm{m}$ isotropic voxels, and provided sufficient resolution to segment the proximal arterial vasculature. The identifiable surface arteries include the communicating arteries of the Circle of Willis (CoW), basilar artery (Bas), and the anterior cerebral artery (ACA) (Figure 4D). The identifiable branching arteries include the posterior cerebral artery (PCA), middle cerebral artery (MCA) and olfactory arteries (OAs) (Figure 4D).

The periarterial spaces were identified based on their unique concentration dynamics and are named periarterial due to their location, surrounding the major arteries. Periarterial spaces surrounding the surface arteries (PAS $\mathrm{Purf}_{\text {Sun }}$ ) and the branching arteries $\left(\mathrm{PAS}_{\mathrm{Branch}}\right)$ (Figure 4B and C) were defined by high contrast concentration ([gadoteridol] $>0.15 \mathrm{mM}$ at $\mathrm{t}=20 \mathrm{~min}$ ) and proximity $\left(<=7\right.$ voxels for $\mathrm{PAS}_{\text {Surf }}$ and $<=3$ voxels for $\left.\mathrm{PAS}_{\text {Branch }}\right)$ to major arteries $\left(\mathrm{T}_{1,0}<1,000\right.$ 1,400 (depending on subject) determined from the baseline (pre-contrast) MRI scans). Periarterial space in the segmented model does not necessarily denote anatomical periarterial space, but correlates well with the structure and width of the preferential transport routes 
characterized by Mestre et al. (18) and Tithof et al. (56) and identified by those groups as PVS surrounding arteries (see Supplementary Materials). In addition, the segmented periarterial volumes of the model serve the purpose of the preferential transport routes identified as periarterial space in the glymphatic model.

At $60 \mu \mathrm{m}$ resolution, smaller arteries and the microvasculature could not be individually resolved from the wider brain interstitium and were thus lumped into voxels of the brain tissue volume (Figure 5). Therefore, $D_{\text {eff }}$ estimated for the brain tissue volume will lump, or combine, interstitial and small periarterial convection as well as diffusion. Contrast transport into the arteries and across the ventricular walls (between the CSF compartment within the ventricles and the surrounding brain tissue) was negligible and these regions were assigned extremely small transport parameters $(0.000001 \%$ of the brain tissue region). Thus, based on the segmentation described above, the current model defines optimal parameters for three key regions in brain-wide transport: the $\mathrm{PAS}_{\text {Surf }}$, the $\mathrm{PAS}_{\mathrm{Branch}}$, and the remaining brain tissue (BT), combining interstitial and small perivascular spaces.

\section{Transport Parameters}

The average $D_{\text {eff }}$ (and standard deviation) for each anatomical subdomain are reported in Table 1. The $D_{e f f}$ for each subdomain is greater than $D_{a p p}$, indicating that transport is faster than attributable to diffusion alone throughout the brain. The estimated value for $D_{e f f}$ is greatest for the regions surrounding the major arterial spaces $\left(\mathrm{PAS}_{\mathrm{Surf}}, \mathrm{PAS}_{\mathrm{Branch}}\right)$ whose values are of similar magnitude. In fact, $D_{e f f}$ in the periarterial regions is so much faster than $D_{a p p}$ $(>10,000 \mathrm{X}$ faster) that periarterial transport can only be explained by convection. Dispersion is predicted to increase the rate of transport over diffusion by a factor of as much as two (20), which would account for only a very small fraction of the observed enhancement. 
Table 1. Quantitative Analysis of Brain Transport Parameters for each Anatomical Subdomain.

\begin{tabular}{|l|c|c|c|c|l|}
\hline \multicolumn{1}{|c|}{$\begin{array}{c}\text { Anatomical } \\
\text { Subdomain }\end{array}$} & $\begin{array}{c}\boldsymbol{D}_{\text {eff }} \\
\left(\mathbf{m m}^{2} / \mathbf{m i n}\right)\end{array}$ & $\begin{array}{c}\text { Peclet } \\
\text { Number }\end{array}$ & $\begin{array}{c}\text { Charac. } \\
\text { Length } \\
(\mathbf{m m})\end{array}$ & $\begin{array}{c}\text { Estimated } \\
\text { Velocity } \\
(\mathbf{m m} / \mathbf{m i n})\end{array}$ & \multicolumn{1}{|c|}{ Published Transport Parameter } \\
\hline $\begin{array}{l}\text { Branching Periarterial } \\
\text { Space (PAS }\end{array}$ & $60 \pm 20$ & 12,000 & 5 & $12 \pm 4$ & $\begin{array}{l}v_{\text {BAS }}=1.2 \mathrm{~mm} / \mathrm{min} \\
(18,19)\end{array}$ \\
\hline $\begin{array}{l}\text { Surface Periarterial } \\
\text { Space (PAS }\end{array}$ & $95 \pm 40$ & 19,000 & 12 & $8 \pm 3$ & $\begin{array}{l}v_{\text {PAS }}=1.2 \mathrm{~mm} / \mathrm{min} \\
(18,19)\end{array}$ \\
\hline $\begin{array}{l}\text { Brain Tissue (BT): } \\
\text { Interstitial space, PVS } \\
\text { of microvessels }\end{array}$ & $0.10 \pm 0.04$ & 18 & 0.5 & $0.2 \pm 0.1$ & $\begin{array}{l}D_{a p p}=0.005 \mathrm{~mm}^{2} / \mathrm{min}_{(46,48)} \\
v_{I S}=0.01 \mathrm{~mm} / \mathrm{min}_{(29)} \\
v_{P A S}=\text { not known }\end{array}$ \\
\hline
\end{tabular}

Table reports: 1) optimal effective diffusivity $\left(D_{e f f}\right)$, 2) Peclet number reflecting the ratio of convective to diffusive transport, 3) average velocity estimated from $D_{\text {eff }}$ using a characteristic length for the anatomical subdomain, and 4) measured brain transport parameters (or parameters estimated using a model) from the literature for comparison. (Error is one standard deviation.)

In the BT subdomain, which combines interstitial space and PVSs associated with smaller arteries and the microcirculation, the enhancement of overall transport $\left(D_{e f f}\right)$ over $D_{a p p}$ is smaller (10-25X), but still supports the presence of convection within the bulk tissue. As discussed above, $D_{\text {eff }}$ of the BT volume represents a combination of diffusion, periarterial convection, periarterial dispersion, and interstitial convection. For a small molecule like gadoteridol ( $\sim 550 \mathrm{Da})$, interstitial convection and diffusion are predicted to have similar rates (29). Dispersion could also enhance transport within the smaller PVSs, however, as discussed above, is unlikely to have a contribution high enough to account for the remaining enhancement of $D_{e f f}$ within the BT subdomain over $D_{a p p}$. Therefore, much of the increase in $D_{e f f}$ over $D_{a p p}$ observed in the BT volume is likely attributable to periarterial convection in PVSs surrounding smaller cerebral blood vessels.

\section{Simulation Comparison}

Figure 6 shows simulated concentration contours compared to concentration calculated from DCE-MRI data for a representative mouse (see Supplemental Material for 3D animations and 2D slices). Contrast agent progression follows the same pattern from its posterior injection site near 
the cisterna magna, as observed in other studies (31-37): 1) contrast moves rapidly along the ventral surface of the brain following surface arteries, reaching the anterior brain in less than 10 min, 2) contrast follows branching arteries into the brain parenchyma, and 3) moves into the surrounding brain tissue from these major periarterial pathways. Given the simplifications applied in the present model, concentration simulations are visually well matched to the experimental data. In the simulation, however, contrast does not move as rapidly towards the anterior brain at early time points and the volume occupied by contrast concentration $>0.1 \mathrm{mM}$ remains greater in the posterior brain compared to the observed experimental distribution. This discrepancy is an outcome of modelling convective transport, which has a first-order (linear) relationship with concentration gradient (Eqn. 1), using a diffusive model, which is second-order (quadratic) with respect to concentration gradient (Eqn. 2). A quadratic model, such as that used in the simulation, will yield higher concentration near the contrast source (the posterior brain) and decrease more rapidly moving anteriorly, while in a linear model, such as that of convection in the full transport equation, concentration will follow a linear decline. The observed first-order relationship between contrast concentration data and rostro-caudal distance is additional evidence in support of periarterial convection.

A more refined inspection of the simulation outcome versus the experimental data (Figure 6 and Supplemental Material) shows that at later time points the experimental data exhibit heterogeneity of contrast concentration (e.g. distinct areas of high and low concentrations), while in the simulation the contrast concentrations decline smoothly through the tissue. This smooth dispersal is the expected outcome of a diffusive model (Eqn. 2). Therefore, such discrepancies are expected given the model chosen and the anatomical simplifications made in this work. 
The heterogeneity of the measured contrast concentration at later time points suggests that the brain tissue is more heterogeneous than modelled, as are the transport mechanisms underlying this anatomical heterogeneity. Certainly, the local agreement between the simulations and the experimental data can be improved by including greater anatomical detail. However, the addition of these details adds complexity and requires more adjustable parameters that dilute the usefulness of the quantified parameters, add new potential sources of error and require extremely large computational resources. Although the model does not perfectly simulate the experimental data, the parameters determined from the model well describe macroscopic transport mechanisms throughout the brain.

\section{Discussion}

The effective diffusivities $\left(D_{e f f}\right)$ reported above are orders of magnitude larger than diffusivity of the contrast agent through brain tissue $\left(D_{a p p}\right)$, and thus indicate that convection is a significant contributor to transport throughout the brain. Optimal agreement between the simulation and DCE-MRI data was obtained with $D_{\text {eff }}>D_{a p p}$ for all modelled brain regions and $D_{e f f} \gg D_{a p p}$ in the periarterial spaces. In this Discussion section, the $D_{\text {eff }}$ results are further analyzed using concepts from transport phenomena and the results of that analysis compared to values reported in literature.

\section{Mechanisms of Transport.}

Péclet number $(\mathrm{Pe})$ is a dimensionless group that assesses the relative importance of convective to diffusive transport rates:

$$
P e=\frac{\text { rate of convection }}{\text { rate of diffusion }}=\frac{L v}{D}=\frac{D_{e f f}-D_{a p p}-D_{\text {disp }}}{D_{a p p}}
$$


If transport is predominantly by diffusion (i.e., by random molecular motion in a stagnant fluid), then $P e \ll 1$, and if transport is predominantly by convection (i.e., by bulk fluid motion), $P e \gg$

1. Table 1 reports $P e$ for each anatomical region using $D_{a p p}=0.005 \mathrm{~mm}^{2} / \mathrm{min}$ (Eqn. 3) and $D_{\text {disp }}=D_{a p p}(20)$. In both periarterial spaces $\left(\mathrm{PAS}_{\text {Surf }}\right.$ and $\left.\mathrm{PAS}_{\mathrm{Branch}}\right), P e$ exceeds 10,000 and convection (or bulk flow) is the dominant mechanism of transport. The brain tissue (BT), which consists of a combination of interstitial tissue and PVSs associated with blood vessels, exhibits an intermediate $P e=18$, where convection and diffusion are both relevant, but convection remains the larger mechanism of transport for the contrast agent. From the Péclet analysis, we conclude convection is present within and significant to transport within the wider brain tissue volume, however the contribution of each of its physiological components (the bulk interstitium versus microvascular PVS) is not determinable. It is also important to note the contrast agent used in this DCE-MRI study is a smaller molecule (gadoteridol, $559 \mathrm{Da}$ ) compared to biological macromolecules, including peptides and proteins of interest in neurodegeneration, such as amyloid $\beta(4.5 \mathrm{kDa})$. These larger molecules have smaller $D_{a p p}$, and therefore larger $P e$, meaning the convection observed here will have an even greater effect on the overall transport of such macromolecules.

\section{Average Velocity.}

Using the definition of Péclet number (Eqn. 4) and a characteristic length for transport in each region, $D_{\text {eff }}$ can be used to estimate average fluid velocity $(v)$, which is reported for each region in Table 1. Periarterial convection is estimated to have an average velocity of $v_{P A S}=8$ $\mathrm{mm} / \mathrm{min}$ in the PAS $\mathrm{Purf}_{\text {and }} v_{P A S}=12 \mathrm{~mm} / \mathrm{min}$ in the $\mathrm{PAS}_{\text {Branch. }}$ The choice of characteristic length has a significant impact on the velocity calculated in this way. The characteristic lengths 
reported in Table 1 for the $\mathrm{PAS}_{\text {Surf }}$ and $\mathrm{PAS}_{\text {Branch }}$ were chosen based on the length dimension of the brain in each direction (caudal-rostral for $\mathrm{PAS}_{\text {Surf }}$ and ventral-dorsal for $\mathrm{PAS}_{\mathrm{Branch}}$ ). As the vasculature followed by the PVS takes a tortuous, branching path and is connected from the major arteries down to the capillaries, the appropriate characteristic length for the periarterial regions may be longer than estimated, which would result in lower velocities. Perivascular fluid velocity at specific locations along the MCA (and its immediate branches) was measured to be $1.2 \mathrm{~mm} / \mathrm{min}$ by both Mestre et al. and Bedussi et al. $(18,19)$. For comparing velocity measured at a specific location to an average velocity estimated over a system, the velocities are similar.

The BT volume represents a combination of interstitial transport and perivascular transport surrounding smaller blood vessels (illustrated in Figures $1 \& 3 \mathrm{~B}$ ). Therefore, literature values are investigated for each and compared to the estimated average velocity of $v_{B T}=0.1-0.3$ $\mathrm{mm} / \mathrm{min}$ (Table 1). In previous work, Ray et al. estimated interstitial flow in the brain (which is expected to be the lowest velocity for all anatomical regions considered here) on the order of $v_{I S}=0.01 \mathrm{~mm} / \mathrm{min}(29)$. Measurements of interstitial flow through tissues outside the brain (in the periphery), which likely represent an upper limit on brain interstitial transport, report $v_{I S}=$ 0.006- $0.12 \mathrm{~mm} / \mathrm{min}(57)$. Both estimates of interstitial velocity are lower by an order of magnitude than the average velocity estimated from the $D_{e f f}$ in the lumped BT volume, indicating the likelihood of a significant contribution from PVS flow.

Another way to think about velocity in the brain tissue region is as the velocity of a transport 'front'. The smaller penetrating arteries and microvessels branch out in many directions, so there may be no clear direction of flow on the scale of the BT volume either in the penetrating periarterial space or for the interstitial flow that runs from periarterial to perivenous space. Instead, contrast progresses more rapidly than by diffusion alone, but along a tortuous 
convective path. Therefore, the velocity calculated in Table 1, $v_{B T}=0.1-0.3 \mathrm{~mm} / \mathrm{min}$, may be better described as the velocity of a 'front' progressing deeper into the brain, as opposed to the velocity of the actual interstitial or periarterial flow. Plog et al. measured front velocities on the order of $0.1-1 \mathrm{~mm} / \mathrm{min}$ in mice in the space between large vessels (analogous to the BT volume in this work) (58). This front velocity, or an enhancement factor of $D_{\text {eff }} / D_{a p p}$, may be a better representation of transport for the combined effects of periarterial convection, interstitial convection, dispersion, and diffusion in the brain tissue.

The results presented above for both the periarterial spaces of major branching arteries (PAS $\mathrm{Branch})$ and the periarterial space of smaller penetrating arteries (included in the BT volume) support continuation of periarterial flow into the brain along penetrating arteries. Mestre et al. (18) and Bedussi et al. (19) reported that 1- $\mu \mathrm{m}$ microspheres tracking fluid movement in the periarterial space surrounding the MCA were excluded from smaller branches of that artery penetrating into the brain. This observation raised the question of whether periarterial flow continues into the brain, and if so, whether the penetrating periarterial spaces are filled with protein networks that behave like a porous media and impede flow. In the model, the PAS $\mathrm{Branch}_{\mathrm{B}}$ is comprised of PVS of the MCA, which resides primarily on the surface of the brain, but also the posterior cerebral artery (PCA) and the OAs, which penetrate into the brain tissue. Convection was apparent along these large arteries of the PAS $\mathrm{Branch}_{\text {at }}$ a magnitude that suggests open channel flow, not flow through porous media. Convection is also supported for the space of small arteries and microvessels. Although the actual flow velocity in the periarterial space of smaller arteries cannot be determined from this analysis performed at the scale of the whole brain, it is clear from the results that such flow significantly enhances transport over diffusion alone. Therefore, the results reported here support open-channel periarterial flow along large 
penetrating arteries and periarterial flow along smaller arteries that may be open-channel or porous media flow.

\section{Sources of Error}

Segmentation. To estimate transport parameters for regions of the brain demonstrating different transport dynamics, the whole-brain model was segmented into volumes defined by either anatomical features determined from pre-contrast images and/or contrast concentration. DCE-MRI measures the average signal for the volume contained in each voxel. Therefore, at the boundaries between segmented volumes a voxel may contain more than one anatomical feature and the signal measured is an average of the signals resulting from each feature. Since the voxel size, or resolution, of $100 \mu \mathrm{m}$ is significant with respect to anatomical features, "mixing" of different tissues at the boundaries of the segmented volumes is unavoidable. For example, there will be some fraction of brain tissue included in the periarterial volumes and vice versa. This "mixing" at the boundaries can be a source of error in estimating separate transport parameters, because there is not a clean division between features and concentration calculated from signal near boundaries includes contributions from each feature. The BT volume is $99.9+\%$ of the total volume (by number of vertices), therefore, any PAS concentration dynamics incorporated into the BT volume at its boundaries are likely to have very little impact on the estimated BT $D_{e f f}$. The estimated $D_{\text {eff }}$ for the $\mathrm{PAS}_{\text {Surf }}$ and $\mathrm{PAS}_{\mathrm{Branch}}$ volumes are significantly greater than the BT $D_{\text {eff }}(>600 \mathrm{Xs})$, therefore, any BT concentration dynamics incorporated into the PAS volumes at the boundaries are also likely to have little impact. In conclusion, although exact segmentation of features is limited by the resolution of the DCE-MRI measurements, such limitations are not 
likely to impact the transport parameter estimates which are significantly different between regions sharing boundaries.

Transport Model. In order to develop a computationally feasible model, the anatomical features of the brain were substantially simplified. Although including additional anatomical subdomains (with unique $D_{\text {eff }}$ ) would have led to improved agreement between simulations and data, it would also have resulted in numerous adjustable parameters that diluted the usefulness of the results. The goal of the present study is to define parameters that describe general transport over the macroscopic scale of the brain, rather than the refined details of specific tissues within the brain, in order to better understand the prevalence and magnitude of convection or dispersion in molecular transport across the entire brain. As such, the simplifications applied to the anatomical framework aid in developing useful transport parameter estimates.

The equation describing transport in the brain was also simplified for this analysis, lumping all transport mechanisms into a single parameter for each region and forcing the mathematical structure of diffusive transport. Careful comparisons of data and simulations show that the relationship between concentration and distance, particularly in the perivascular space, better follows the mathematical form of convection. The model could be greatly improved by specifically including all mechanisms of transport as shown in Equation 1. However, to calculate convection, a driving force, such as pressure, must be known throughout the brain, which it is not. In addition, convection is a vector, which has a magnitude and a direction, adding a great deal of complexity. Alternatively, a convective field could be measured experimentally, possibly by Intravoxel Incoherent Motion (IVIM) MRI (59). The velocity field could be applied to the finite-element mesh directly and the transport model could be used to answer more subtle questions about brain transport, i.e. modelling disease states. 
The transport model did not include efflux routes for the contrast agent to exit the brain volume. In the glymphatic model, fluid and molecules exit the brain tissue through the perivenous space, ultimately emptying to extracranial lymphatic vessels outside the blood-brain barrier (8). Glymphatic efflux routes are not well understood and are therefore a place of continued investigation $(22,25)$. If efflux was significant to transport over the time scale of the experiments, the total amount of tracer in the brain would be expected to decrease steadily after the injection was complete. However, calculations show increasing total amounts of Gad over the course of the experiment, which supports that efflux is not a significant contributor to transport over the time scale investigated. As more clarity is gained about mechanisms of efflux, distributed efflux or specific routes may be added to the model to improve its representation of the physical situation and allow analysis of longer time-series data.

DCE-MRI Experiments. Even though it is a powerful experimental tool, MRI is an inherently noisy technique with a low signal-to-noise ratio. In live biological subjects, this noise is exacerbated by small movements in the tissue, such as blood pumping through vessels, or movement of the entire subject. MRI data is often filtered to reduce noise and smooth the data, but at the cost of averaging the data over regions considered large relative to anatomical details. In order to investigate the interaction of transport with specific anatomical features, the decision was made to leave the data unfiltered. (Some minimal Gaussian filtering occurred as a result of interpolating the MRI data onto the finite-element mesh.) A consequence of using the unfiltered data is greater variability between points in space and time, leading to greater error between the simulation and the data. However, all parameter combinations experience this same noise, and the transport parameters giving the minimum difference between simulation and data, which is relative, should not be greatly affected. 
The DCE-MRI data used in this analysis exhibited T2/T2* interference at high contrast concentration, sacrificing useful data near the injection site. The DCE-MRI experimental parameters (i.e., pulse sequence) were optimized for measuring low concentrations of gadoteridol in order to follow it deep into the tissue and possibly elucidate efflux routes. In particular, $\mathrm{T} 2 / \mathrm{T} 2 *$ interference obscured the rapid transport path from the injection site to the tissue surrounding the ventral surface arteries, adding uncertainty to the $\mathrm{PAS}_{\text {surf }}$ geometry used in the model. T2/T2* interference was more likely to increase variability than to result in higher or lower values for $D_{e f f}$.

It has been questioned whether the contrast infusion, during the first 20 minutes of the experiments used here, might induce convection (26). Raghunandan et al. directly addressed this question by comparing a tracer study with traditional infusion techniques, where tracer solution is slowly injected into the CSF, to a study where CSF was removed from the cranium at the same rate the tracer solution was injected, for no net addition of fluid (60). The experimental results, where periarterial velocity in mice was measured using the same methods as Mestre et al. (18), were statistically identical for the two cases. The infusion rate used by Raghunandan et al. is four times higher than the infusion rate for the DCE-MRI experiments reported here. In addition, Bedussi et al. (19) observed no increase in intracranial pressure (ICP) (CSF pressure remained within the range of pre-infusion ICP) at $70 \%$ of the infusion rate used in the experiments reported here. Therefore, published experimental results support that, at the infusion rate used for the DCE-MRI experiments analyzed here, convection is not an artifact of tracer infusion.

The model developed in this work could also aid in assessing this concern, but analysis of the current data is limited by $\mathrm{T} 2 / \mathrm{T} 2 *$ interference in the important region near the injection site 
and few data sets during the infusion. Even with those limitations, a simulation fitting different $D_{\text {eff }}$ parameter sets during and after infusion showed potential differences, but still indicated significant convection in the PVSs surrounding major arteries and transport that is faster than diffusion alone in the brain tissue region post infusion.

\section{System Efficiency.}

Finally, we analyze the transport parameter values as an integrated system. Constructal Theory, which has been used to analyze the efficiency of biological systems $(61,62)$, states natural systems optimize over time and optimize around the constraints of the space allocated to the 'function of the system', the functional space. In an optimized natural system, transport across the functional space will be the rate limiting step, i.e., have the longest characteristic transport time $(\tau)$ in the system, and the remaining 'flow system', will optimize around the functional space. For the glymphatic system, the functional space is the interstitial space, where nutrients are delivered to the cells and waste products are carried away, while the PVS makes up the flow system. Using $D_{e f f}$ and characteristic lengths discussed above, $\tau$ (interstitial space) is about 3 minutes for small molecules (100 Da) and 13 minutes for large molecules (1,000 Da), while $\tau($ major periarterial space $)=1-3 \min$ and $\tau($ minor periarterial space $)=2-5 \mathrm{~min}$. For transport of small molecules, $\tau$ is similar for each 'step', indicating the whole system is optimized. For transport of large molecules, the interstitial space is rate limiting as predicted, likely limited by the inherent slow diffusivity of large molecules and constrained by the maximum flow velocity tolerated by brain cells, and the flow system is faster.

\section{Conclusions.}


Quantitative analysis of DCE-MRI data for the whole mouse brain using a finite-element transport model, indicates convection is present throughout the brain. Convection is estimated to be dominant in the periarterial space of major surface and branching arteries, where $P e>10,000$. Importantly, convection was predicted to continue into the brain tissue, demonstrating that it is more than a surface phenomenon. Periarterial convection for smaller penetrating arteries could not be separated from interstitial convection as the data lacked the resolution to make this distinction. However, comparison to estimated and upper bound values for interstitial flow suggests significant convection in the PVS throughout the dispersed brain tissue.

The whole-brain transport model described in this work is structured for continuous improvement as new information comes to light and may also be appropriately connected with small-scale models, such as for interstitial transport (29), to develop a multi-scale transport model. The transport analysis described here could be improved with experimental data investigating molecules of different sizes, i.e. $500 \mathrm{Da}$ and 10,000 Da. Diffusion and dispersion are dependent on molecular size, while convection is relatively independent of molecular size. Therefore, comparison of transport data and parameters from contrast agents of significantly different molecular size would provide further evidence towards convective versus diffusive or dispersive transport.

\section{Additional files}

Additional file 1. 3D animations of concentration data and representative simulation. Additional file 2. Supplemental Material consisting of: 2D slices of concentration data and representative simulation, pre-contrast images and tissue properties, determination of contrast-agent relaxivity, example error contours for determining optimal transport parameters, periarterial-width sensitivity analysis and comparison to literature, and example FEniCS/python code for transport model and simulation.

\section{Abbreviations}

ACA: anterior cerebral artery; Bas: basilar artery; BT: brain tissue (combined interstitial space and perivascular space of smaller arteries); CoW: Circle of Willis; CSF: cerebrospinal fluid; DCE-MRI: dynamic contrast-enhanced MRI; $D_{a p p}$ : apparent diffusivity; $D_{\text {disp }}$ : dispersion coefficient; $D_{\text {eff: }}$ effective diffusivity; FEM: finite-element model; MCA: middle cerebral artery; MRI: magnetic resonance imaging; OA: olfactory artery; PAS: surface periarterial space; PCA: posterior cerebral artery; Pe: Péclet number; PVS: perivascular space; $r m s$ : root mean square error; $v_{I S}$ : interstitial superficial velocity; $v_{P A S}$ : periarterial space velocity. 
Authors' contributions

JH and LR conceived and designed the study; LR performed the simulations and analyzed the results; MS and MP performed DCE-MRI experiments and executed post-processing and registration of DCE-MRI data; MP provided valuable knowledge regarding MRI experiments and data; JI provided valuable knowledge regarding neurophysiology; LR wrote the article with input from the other authors. All authors read, edited, and approved the final manuscript.

\section{Competing interests}

The authors declare that they have no competing interests.

\section{Availability of data and materials}

The datasets and computer code supporting the conclusions of this article are available from the corresponding author on reasonable request. An addition, an example code is provided in the Supplemental Materials.

\section{Consent for publication}

Not applicable.

Ethics approval and consent to participate

Not applicable.

Funding

JH and LR were partially supported by the NSF (DMS-1361240). 


\section{References}

1. Nicholson C, Hrabetova S. Brain Extracellular Space: The Final Frontier of Neuroscience. Biophysical Journal. 2017;113(10):2133-42. DOI: 10.1016/j.bpj.2017.06.052.

2. Selkoe DJ, Hardy J. The amyloid hypothesis of Alzheimer's disease at 25years. Embo Molecular Medicine. 2016;8(6):595-608. DOI: 10.15252/emmm.201606210.

3. Iliff JJ, Chen MJ, Plog BA, Zeppenfeld DM, Soltero M, Yang L, et al. Impairment of Glymphatic Pathway Function Promotes Tau Pathology after Traumatic Brain Injury. J Neurosci. 2014;34(49):16180-93. DOI: 10.1523/jneurosci.3020-14.2014.

4. Nedergaard M, Goldman SA. Glymphatic failure as a final common pathway to dementia. Science. 2020;370(6512):50-+. DOI: 10.1126/science.abb8739.

5. Nicholson C. Diffusion and related transport mechanisms in brain tissue. Reports on Progress in Physics. 2001;64(7):815-84. DOI: 10.1088/0034-4885/64/7/202.

6. Rennels ML, Gregory TF, Blaumanis OR, Fujimoto K, Grady PA. EVIDENCE FOR A PARAVASCULAR FLUID CIRCULATION IN THE MAMMALIAN CENTRAL NERVOUS SYSTEM, PROVIDED BY THE RAPID DISTRIBUTION OF TRACER PROTEIN THROUGHOUT THE BRAIN FROM THE SUBARACHNOID SPACE. Brain Research. 1985;326(1):47-63. DOI: 10.1016/0006-8993(85)91383-6.

7. Cserr HF, Depasquale M, Nicholson C, Patlak CS, Pettigrew KD, Rice ME. EXTRACELLULAR VOLUME DECREASES WHILE CELL-VOLUME IS MAINTAINED BY ION UPTAKE IN RAT-BRAIN DURING ACUTE HYPERNATREMIA. Journal of Physiology-London. 1991;442:277-95. DOI: 10.1113/jphysiol.1991.sp018793.

8. Iliff JJ, Wang M, Liao Y, Plogg BA, Peng W, Gundersen GA, et al. A Paravascular Pathway Facilitates CSF Flow Through the Brain Parenchyma and the Clearance of Interstitial Solutes, Including Amyloid beta. Science Translational Medicine. 2012;4(147). DOI: 10.1126/scitranslmed.3003748.

9. Iliff JJ, Wang M, Zeppenfeld DM, Venkataraman A, Plog BA, Liao Y, et al. Cerebral Arterial Pulsation Drives Paravascular CSF-Interstitial Fluid Exchange in the Murine Brain. J Neurosci. 2013;33(46):18190-9. DOI: 10.1523/jneurosci.1592-13.2013.

10. Xie L, Kang H, Xu Q, Chen MJ, Liao Y, Thiyagarajan M, et al. Sleep Drives Metabolite Clearance from the Adult Brain. Science. 2013;342(6156):373-7. DOI:

10.1126/science. 1241224.

11. Louveau A, Plog BA, Antila S, Alitalo K, Nedergaard M, Kipnis J. Understanding the functions and relationships of the glymphatic system and meningeal lymphatics. Journal of Clinical Investigation. 2017;127(9):3210-9. DOI: 10.1172/jci90603.

12. Aspelund A, Antila S, Proulx ST, Karlsen TV, Karaman S, Detmar M, et al. A dural lymphatic vascular system that drains brain interstitial fluid and macromolecules. Journal of Experimental Medicine. 2015;212(7):991-9. DOI: 10.1084/jem.20142290.

13. Nedergaard M. Garbage Truck of the Brain. Science. 2013;340(6140):1529-30. DOI: 10.1126/science. 1240514 .

14. Abbott NJ, Pizzo ME, Preston JE, Janigro D, Thorne RG. The role of brain barriers in fluid movement in the CNS: is there a 'glymphatic' system? Acta Neuropathologica. 2018;135(3):387-407. DOI: 10.1007/s00401-018-1812-4.

15. Hladky SB, Barrand MA. Mechanisms of fluid movement into, through and out of the brain: evaluation of the evidence. Fluids and Barriers of the Cns. 2014;11. DOI: 10.1186/20458118-11-26. 
16. Smith AJ, Verkman AS. The "glymphatic" mechanism for solute clearance in Alzheimer's disease: game changer or unproven speculation? Faseb Journal. 2018;32(2):543-51. DOI: 10.1096/fj.201700999.

17. Mestre H, Hablitz LM, Xavier ALR, Feng W, Zou W, Pu T, et al. Aquaporin-4dependent glymphatic solute transport in the rodent brain. Elife. 2018;7. DOI:

10.7554/eLife.40070.

18. Mestre H, Tithof J, Du T, Song W, Peng WG, Sweeney AM, et al. Flow of cerebrospinal fluid is driven by arterial pulsations and is reduced in hypertension. Nature Communications. 2018;9. DOI: 10.1038/s41467-018-07318-3.

19. Bedussi B, Almasian M, de Vos J, VanBavel E, Bakker E. Paravascular spaces at the brain surface: Low resistance pathways for cerebrospinal fluid flow. J Cereb Blood Flow Metab. 2018;38(4):719-26. DOI: 10.1177/0271678x17737984.

20. Asgari M, de Zelicourt D, Kurtcuoglu V. Glymphatic solute transport does not require bulk flow. Scientific Reports. 2016;6. DOI: 10.1038/srep38635.

21. Rasmussen MK, Mestre H, Nedergaard M. The glymphatic pathway in neurological disorders. Lancet Neurology. 2018;17(11):1016-24. DOI: 10.1016/s1474-4422(18)30318-1.

22. Benveniste H, Liu X, Koundal S, Sanggaard S, Lee H, Wardlaw J. The Glymphatic System and Waste Clearance with Brain Aging: A Review. Gerontology. 2019;65(2):106-19. DOI: $10.1159 / 000490349$.

23. Rivas FM, Liu J, Martell BC, Du T, Mestre H, Nedergaard M, et al. Surface periarterial spaces of the mouse brain are open, not porous. Journal of the Royal Society Interface. 2020;17(172). DOI: 10.1098/rsif.2020.0593.

24. Bilston LE, Fletcher, David F., Brodbelt, Andrew R., Stoodley, Marcus A. Arterial Pulsation-driven Cerecrospinal Fluid Flow in the Perivascular Space: A Computational Model. Computer Methods in Biomechanics and Biomedical Engineeering. 2003;6(4):235-41.

25. Ray LA, Heys JJ. Fluid Flow and Mass Transport in Brain Tissue. Fluids. 2019;4(4). DOI: 10.3390/fluids4040196.

26. Faghih MM, Keith Sharp M. Mechanisms of tracer transport in cerebral perivascular spaces. Journal of biomechanics. 2021;118:110278-. DOI: 10.1016/j.jbiomech.2021.110278.

27. Holter KE, Kehlet B, Devor A, Sejnowski TJ, Dale AM, Omholt SW, et al. Interstitial solute transport in 3D reconstructed neuropil occurs by diffusion rather than bulk flow. Proceedings of the National Academy of Sciences of the United States of America. 2017;114(37):9894-9. DOI: 10.1073/pnas.1706942114.

28. Jin B-J, Smith AJ, Verkman AS. Spatial model of convective solute transport in brain extracellular space does not support a "glymphatic" mechanism. Journal of General Physiology. 2016;148(6):489-501. DOI: 10.1085/jgp.201611684.

29. Ray L, Iliff JJ, Heys JJ. Analysis of convective and diffusive transport in the brain interstitium. Fluids and Barriers of the CNS. 2019;16(1):6. DOI: 10.1186/s12987-019-0126-9. 30. Benveniste H, Lee H, Ozturk B, Chen X, Koundal S, Vaska P, et al. Glymphatic Cerebrospinal Fluid and Solute Transport Quantified by MRI and PET Imaging. Neuroscience. 2020. DOI: 10.1016/j.neuroscience.2020.11.014.

31. Iliff JJ, Lee H, Yu M, Feng T, Logan J, Nedergaard M, et al. Brain-wide pathway for waste clearance captured by contrast-enhanced MRI. Journal of Clinical Investigation. 2013;123(3):1299-309. DOI: 10.1172/jci67677. 
32. Benveniste H, Heerdt PM, Fontes M, Rothman DL, Volkow ND. Glymphatic System Function in Relation to Anesthesia and Sleep States. Anesthesia and Analgesia. 2019;128(4):747-58. DOI: 10.1213/ane.0000000000004069.

33. Gakuba C, Gaberel T, Goursaud S, Bourges J, Di Palma C, Quenault A, et al. General Anesthesia Inhibits the Activity of the "Glymphatic System". Theranostics. 2018;8(3):710-22. DOI: $10.7150 /$ thno.19154.

34. Dobson H, Sharp MM, Cumpsty R, Criswell TP, Wellman T, Finucane C, et al. The perivascular pathways for influx of cerebrospinal fluid are most efficient in the midbrain. Clinical Science. 2017;131(22):2745-52. DOI: 10.1042/cs20171265.

35. Goulay R, Flament J, Gauberti M, Naveau M, Pasquet N, Gakuba C, et al. Subarachnoid Hemorrhage Severely Impairs Brain Parenchymal Cerebrospinal Fluid Circulation in Nonhuman Primate. Stroke. 2017;48(8):2301-+. DOI: 10.1161/strokeaha.117.017014.

36. Jiang Q, Zhang L, Ding GL, Davoodi-Bojd E, Li QJ, Li L, et al. Impairment of the glymphatic system after diabetes. J Cereb Blood Flow Metab. 2017;37(4):1326-37. DOI: $10.1177 / 0271678 \times 16654702$.

37. Zhou Y, Cai JS, Zhang WH, Gong XX, Yan SQ, Zhang KM, et al. Impairment of the Glymphatic Pathway and Putative Meningeal Lymphatic Vessels in the Aging Human. Annals of Neurology. 2020;87(3):357-69. DOI: 10.1002/ana.25670.

38. Pizzo ME, Wolak DJ, Kumar NN, Brunette E, Brunnquell CL, Hannocks M-J, et al. Intrathecal antibody distribution in the rat brain: surface diffusion, perivascular transport and osmotic enhancement of delivery. Journal of Physiology-London. 2018;596(3):445-75. DOI: 10.1113/jp275105.

39. Koundal S, Elkin R, Nadeem S, Xue YC, Constantinou S, Sanggaard S, et al. Optimal Mass Transport with Lagrangian Workflow Reveals Advective and Diffusion Driven Solute Transport in the Glymphatic System (vol 10, 1990, 2020). Scientific Reports. 2020;10(1). DOI: 10.1038/s41598-020-60586-2.

40. Valnes LM, Mitusch SK, Ringstad G, Eide PK, Funke SW, Mardal K-A. Apparent diffusion coefficient estimates based on 24 hours tracer movement support glymphatic transport in human cerebral cortex. Scientific Reports. 2020;10(1):9176. DOI: 10.1038/s41598-02066042-5.

41. Ringstad G, Valnes LM, Dale AM, Pripp AH, Vatnehol SAS, Emblem KE, et al. Brainwide glymphatic enhancement and clearance in humans assessed with MRI. Jci Insight. 2018;3(13). DOI: 10.1172/jci.insight.121537.

42. Ringstad G, Vatnehol SAS, Eide PK. Glymphatic MRI in idiopathic normal pressure hydrocephalus. Brain. 2017;140:2691-705. DOI: 10.1093/brain/awx191.

43. Buckley DL, Parker GJM. Measuring Contrast Agent Concentration in T1-Weighted Dynamic Contrast-Enhanced MRI. In: Jackson A, Buckley DL, Parker GJM, editors. Dynamic Contrast-Enhanced Magnetic Resonance Imaging in Oncology. Berlin, Heidelberg: Springer Berlin Heidelberg; 2005. p. 69-79.

44. Szomolanyi P, Rohrer M, Frenzel T, Noebauer-Hohmann IM, Jost G, Endrikat J, et al. Comparison of the Relaxivities of Macrocyclic Gadolinium-Based Contrast Agents in Human Plasma at 1.5, 3, and 7 T, and Blood at 3 T. Investigative Radiology. 2019;54(9):559-64. DOI: 10.1097/rli.0000000000000577.

45. Sandmann C, Hodneland E, Modersitzki J. A practical guideline for T-1 reconstruction from various flip angles in MRI. Journal of Algorithms \& Computational Technology. 2016;10(4):213-23. DOI: 10.1177/1748301816656288. 
46. Sykova E, Nicholson C. Diffusion in brain extracellular space. Physiological Reviews. 2008;88(4):1277-340. DOI: 10.1152/physrev.00027.2007.

47. Linninger AA, Somayaji MR, Erickson T, Guo X, Penn RD. Computational methods for predicting drug transport in anisotropic and heterogeneous brain tissue. Journal of Biomechanics. 2008;41(10):2176-87. DOI: 10.1016/j.jbiomech.2008.04.025.

48. Rattanakijsuntorn K, Penkova A, Sadhal SS, Iop. Mass diffusion coefficient measurement for vitreous humor using FEM and MRI. 8th Tsme-International Conference on Mechanical Engineering. IOP Conference Series-Materials Science and Engineering. 2972018.

49. Rorden C. MRIcroS. MATLAB Central File Exchange: Neuroimaging Tools and Resources Collabortory.

50. Cignoni P, Callieri M, Corsini M, Dellepiane M, Ganovelli F, Ranzuglia G. MeshLab: 3D triangular meshes processing and editing. Astrophysics Source Code Library. 2017.

51. Geuzaine C, Remacle J-F. Gmsh: A 3-D finite element mesh generator with built-in preand post-processing facilities. International Journal for Numerical Methods in Engineering. 2009;79(11):1309-31. DOI: 10.1002/nme.2579.

52. Logg AM, KA; Wells, GN. Automated solution of differential equations by the finite element method: the FEniCS book. Berlin: Springer; 2012.

53. Logg AW, GN; Hake, j. DOLFIN: a C++/Python finite element library. Lecture notes in computational science and engineering. In: Automated solution of differential equations by finite element method. Berlin: Springer; 2012.

54. Van Rossum GD, FL. Python 3 Reference Manual. Scotts Valley, CA: CreateSpace; 2009.

55. Ahrens JG, B: Law, C. Paraview: An ENd-User Tool for Large Data Visualization, Visualization Handbook: Elsevier; 2005.

56. Tithof J, Kelley DH, Mestre H, Nedergaard M, Thomas JH. Hydraulic resistance of periarterial spaces in the brain. Fluids and Barriers of the Cns. 2019;16. DOI: 10.1186/s 12987019-0140-y.

57. Swartz MA, Fleury ME. Interstitial flow and its effects in soft tissues. Annual Review of Biomedical Engineering. 2007;9:229-56. DOI: 10.1146/annure/bioeng.9.060906.151850.

58. Plog BA, Mestre H, Olveda GE, Sweeney AM, Kenney HM, Cove A, et al. Transcranial optical imaging reveals a pathway for optimizing the delivery of immunotherapeutics to the brain. Jci Insight. 2018;3(20). DOI: 10.1172/jci.insight.120922.

59. Paschoal AM, Leoni RF, Dos Santos AC, Paiva FF. Intravoxel incoherent motion MRI in neurological and cerebrovascular diseases. Neuroimage Clin. 2018;20:705-14. DOI: 10.1016/j.nicl.2018.08.030.

60. Raghunandan A, Ladron-de-Guevara A, Tithof J, Mestre H, Du T, Nedergaard M, et al. Bulk flow of cerebrospinal fluid observed in periarterial spaces is not an artifact of injection. Elife. 2021;10. DOI: 10.7554/eLife.65958.

61. Bejan A, Lorente S. Constructal law of design and evolution: Physics, biology, technology, and society. Journal of Applied Physics. 2013;113(15). DOI: 10.1063/1.4798429.

62. Reis AH, Miguel AF, Aydin M. Constructal theory of flow architecture of the lungs. Medical Physics. 2004;31(5):1135-40. DOI: 10.1118/1.1705443. 


\section{Figures}

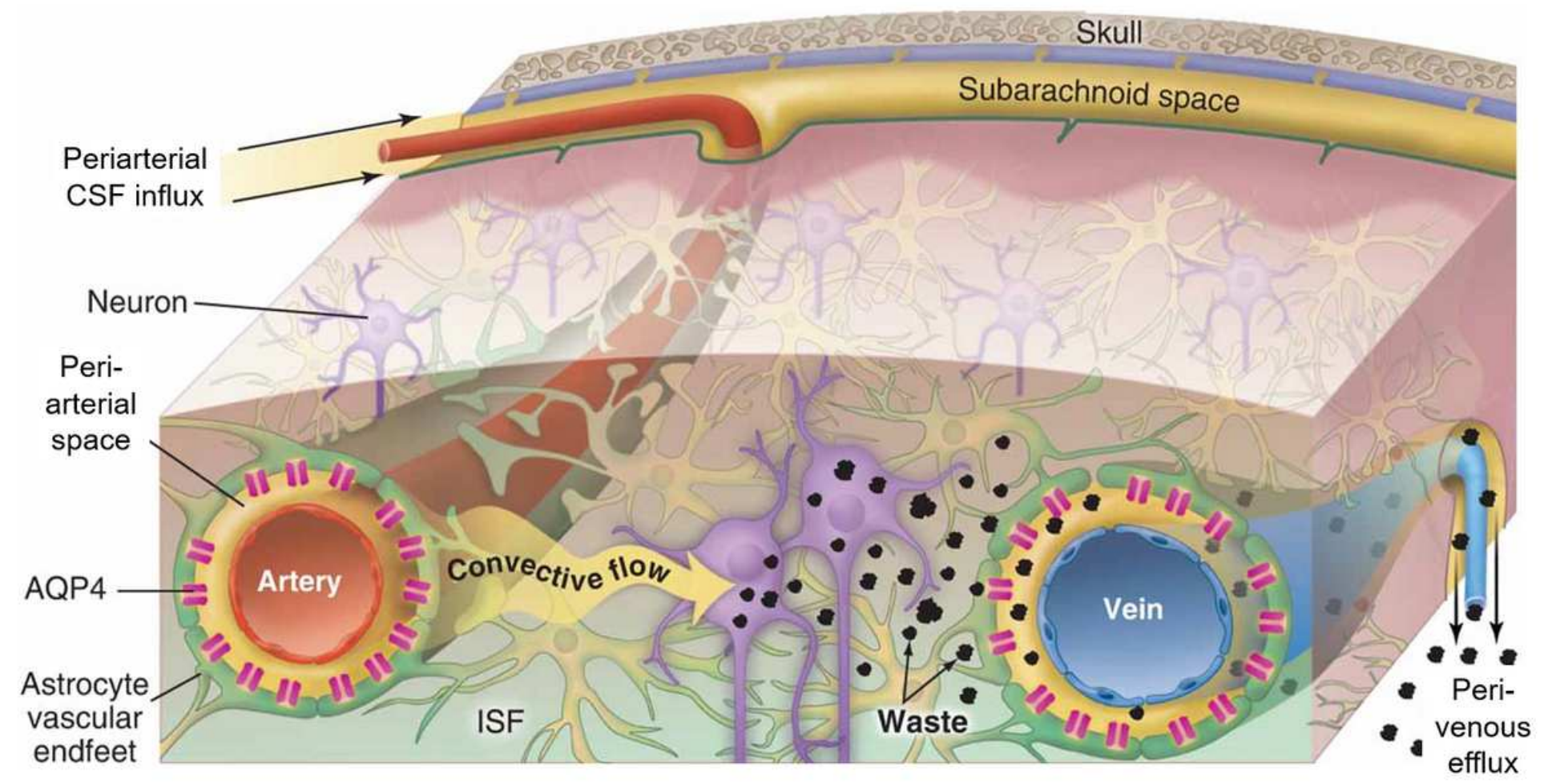

\section{Figure 1}

Perivascular glymphatic transport in the brain. Schematic depicting the proposed mechanism underlying molecular transport in brain tissue, the glymphatic system. Perivascular spaces (PVS) surround cerebral blood vessels and are bounded by the vascular wall on the inside and by the 'endfeet' of astrocyte cells (green) on the outside. It is proposed that cerebrospinal fluid moves inward from the subarachnoid space along periarterial spaces surrounding penetrating arteries deep into the brain. The fluid enters the interstitium through gaps between astroglial endfeet. Interstitial solutes ('waste') are cleared by interstitial convection towards peri-venous spaces and extracranial efflux routes. Perivascular CSF influx and interstitial solute efflux is facilitated by astroglial aquaporin-4 (AQP4) water channels. Reproduced from (13) with nomenclature updated from 'para-arterial' to 'periarterial'. 


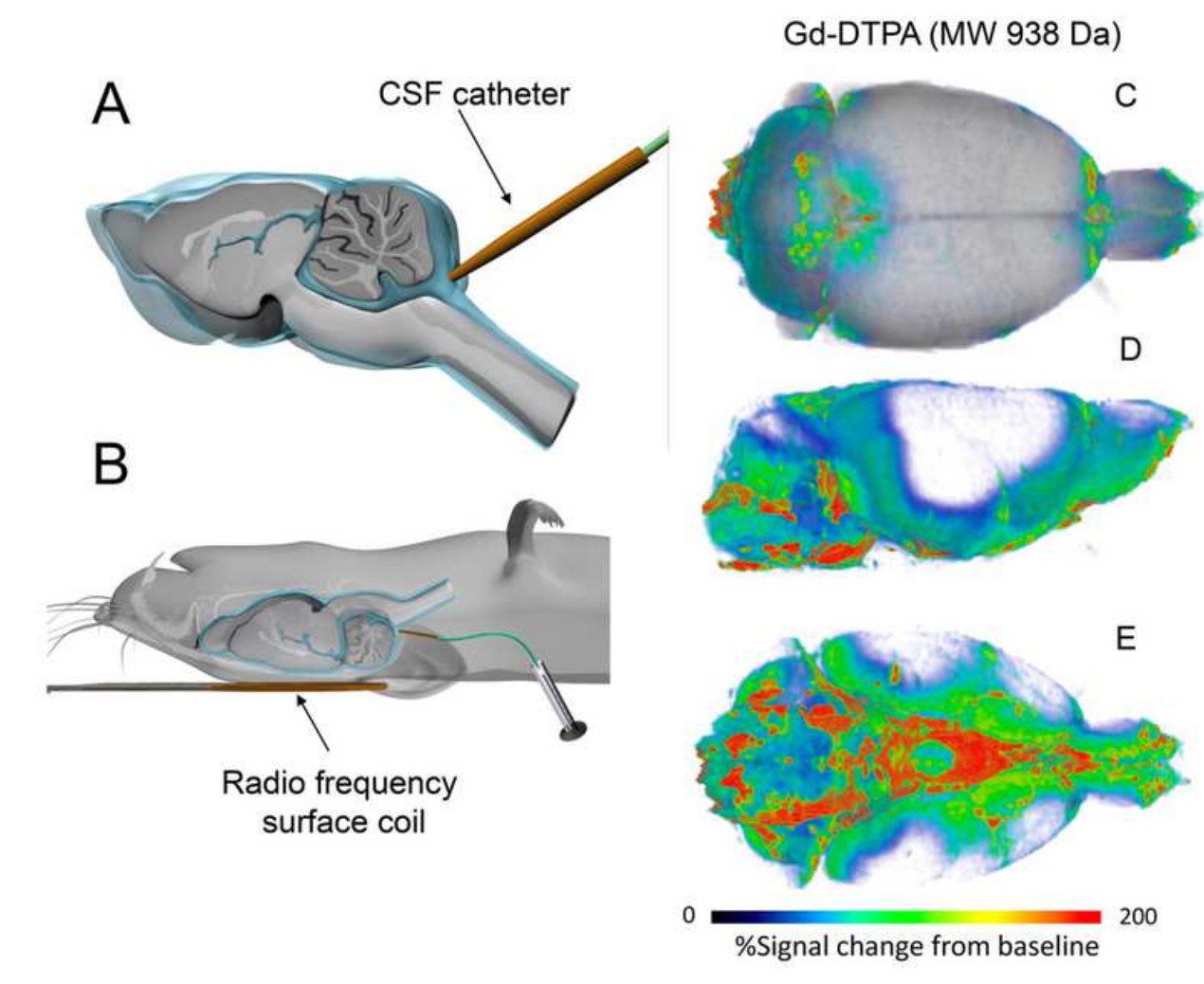

\%Signal change from baseline
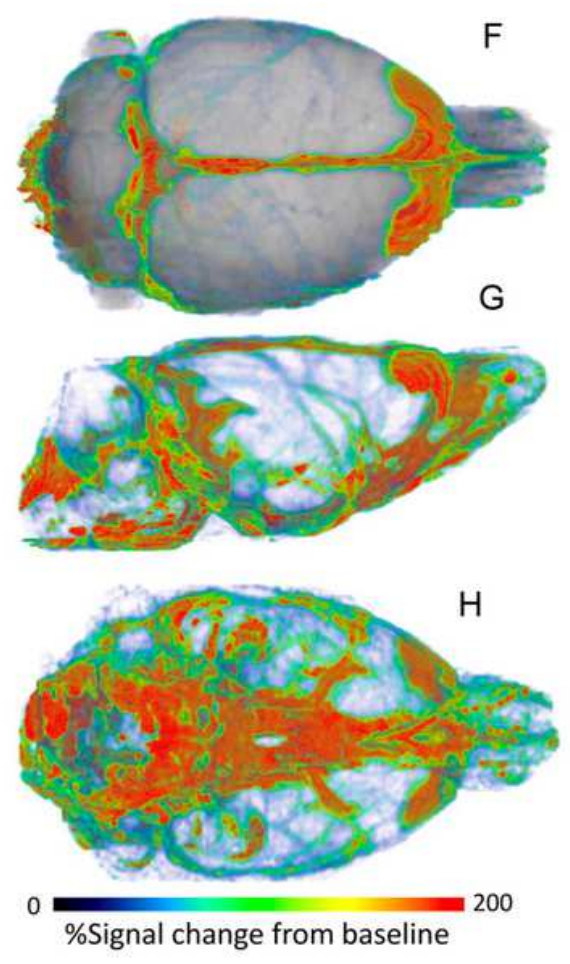

\section{Figure 2}

Overview of dynamic contrast enhanced magnetic resonance imaging (DCE-MRI) following intrathecal gadolinium-based contrast agent injection. (A) Illustration of catheter position for contrast agent delivery into cerebrospinal fluid (CSF, blue liquid) via the cisterna magna of a rat brain. The rat brain is shown in a sagittal plane to show the CSF compartment (blue) in relation to the brain tissue (grey). (B) In preparation for DCE-MRI assessment of glymphatic transport, the anesthetized rat with the indwelling CSF catheter is positioned supine with the small radio frequency surface coil placed under the head. (C-E) 3D volume rendered DCE-MRI images of a rat brain in top (C), lateral decubitus (D) and caudal/ventral view (E) are shown $₫ 90$ min after intrathecal administration of Gd-DTPA (molecular weight, (MW) 938Da). The DCEMRI data has been processed so that the color-coded map represents '\% signal change from baseline'. Red and blue colors represent high and low levels of Gd-DTPA, respectively. There is high glymphatic uptake of Gd-DTPA into the cerebellum, ventral hippocampus, and olfactory bulb. $(F-H)$ 3D volume rendered DCE-MRI of another rat $\triangle 90 \mathrm{~min}$ after CSF administration of GadoSpin which is a large molecule (MW 200,000 Da) compared to Gd-DTPA. Reproduced from (30). 


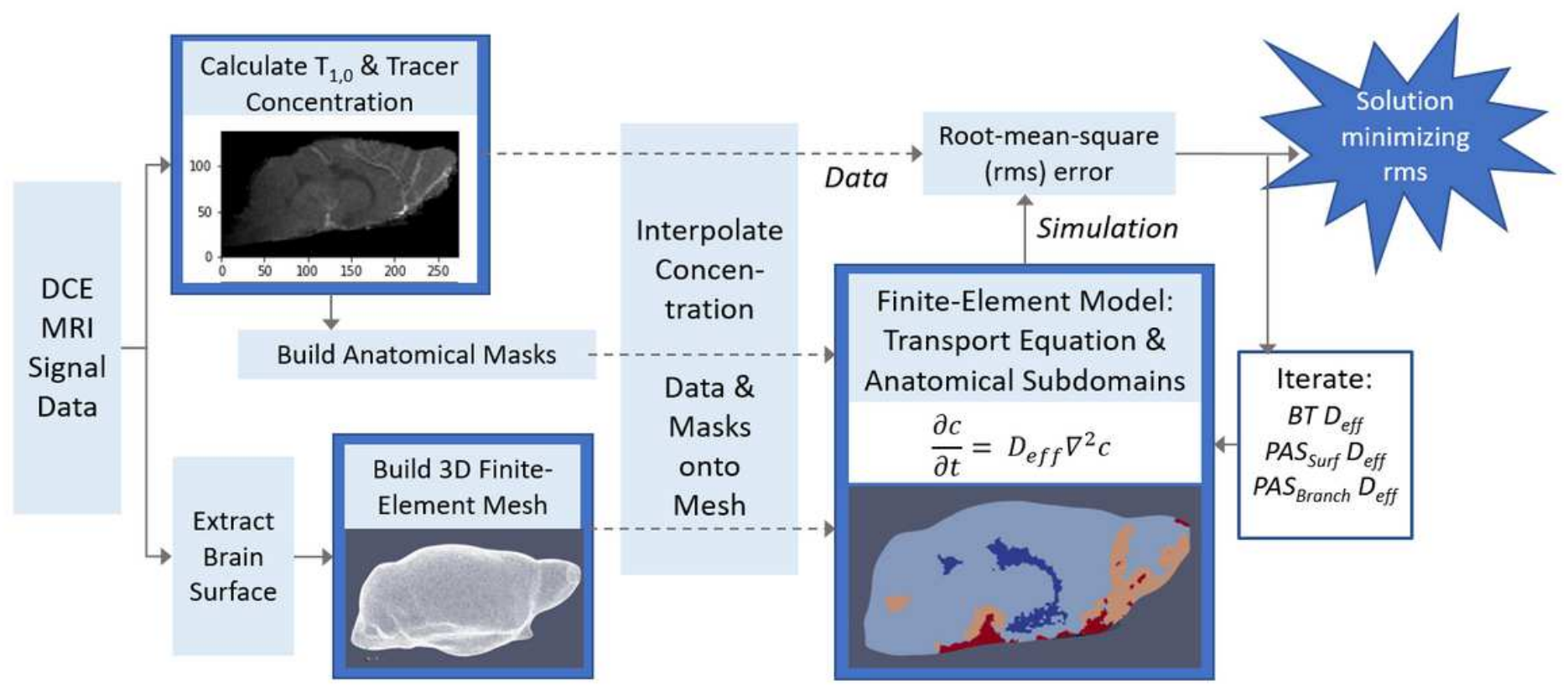

Figure 3

Schematic of brain transport analysis using finite-element modeling with DCE-MRI Data. DCE-MRI signal data is used to calculate contrast agent concentration and T_1,0, a parameter used to identify different types of tissues. Contrast concentration and $T_{-} 1,0$ are used to define anatomical masks for regions relevant to brain-wide transport, including the ventricular system, the macroscopic arterial vasculature, and its associated perivascular spaces. DCE-MRI data is also utilized to extract the surface of the brain. The anatomical masks are unique to each experimental subject. A 3D tetrahedral mesh is built from the brain surface. The concentration data and anatomical masks are interpolated onto the mesh. A finiteelement model is developed utilizing the simplified mass transport equation (Eqn. 2) and subdomains defined by the anatomical masks (Figure 3). Unique effective diffusivities are applied to each subdomain. Simulations are performed for varying effective diffusivities to find the combination of effective diffusivities that minimizes the difference between the concentrations data and the simulation. 

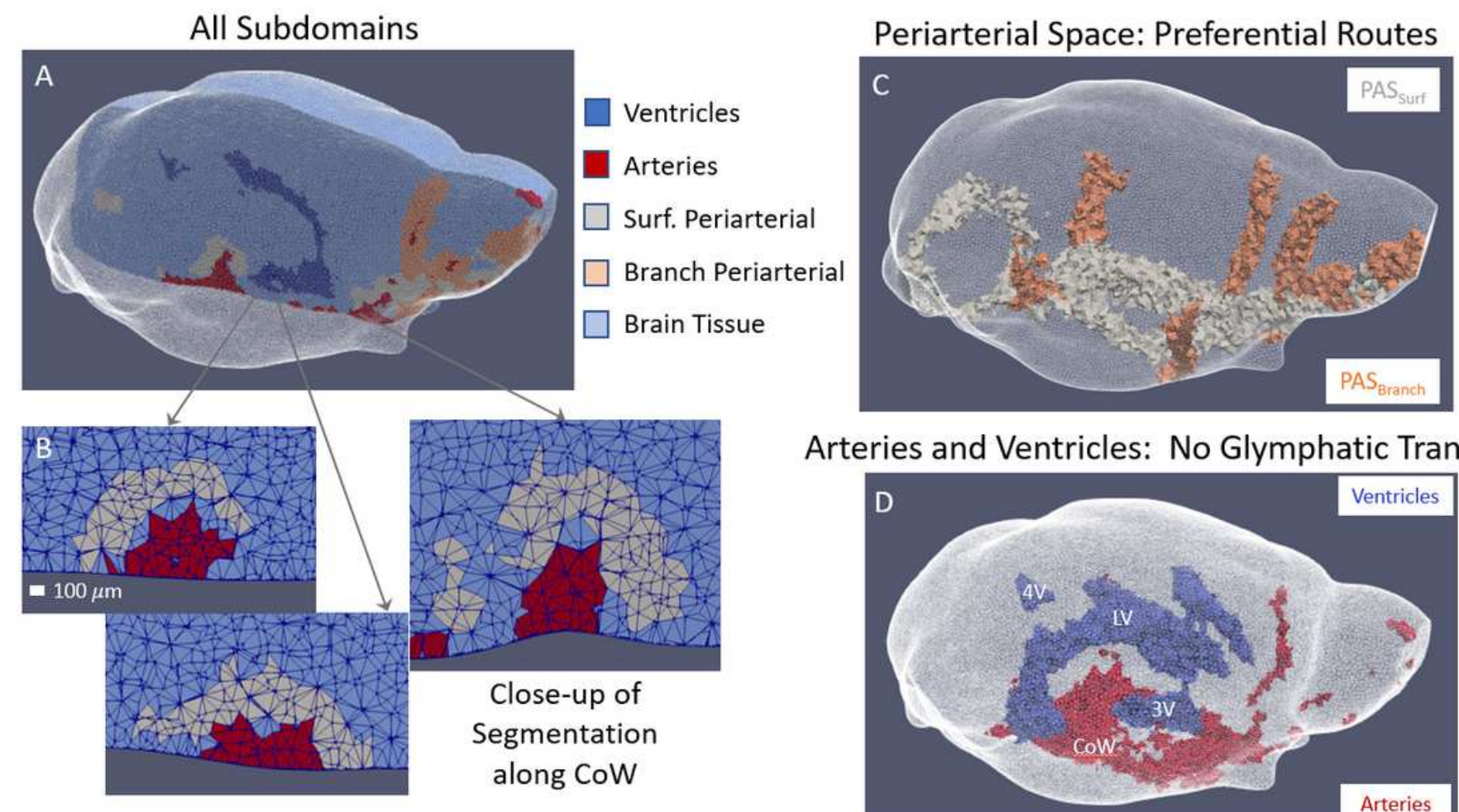

Arteries and Ventricles: No Glymphatic Transport

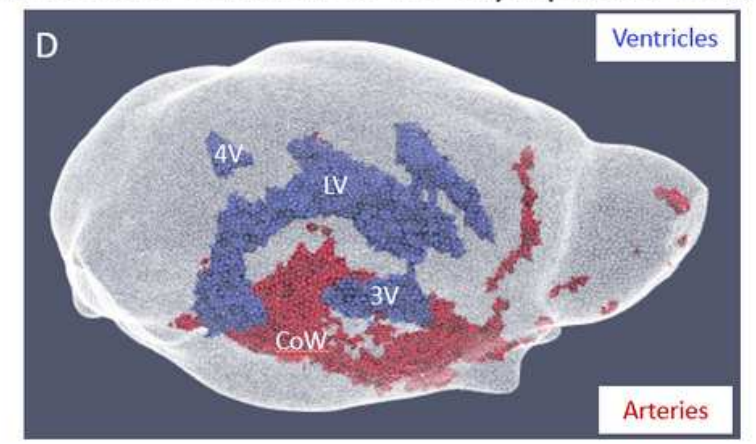

\section{Figure 4}

Anatomical details extracted from MRI data define transport model subdomains. Structure MRI scans and DCE-MRI data were used to build subdomains within the whole-brain finite-element model. Data were analyzed to determine transport parameters for each subdomain within the whole-brain model. (A) Subject-specific, 3D tetrahedral mesh of mouse brain in translucent white (crystal brain). Sagittal crosssection shows all five anatomical subdomains within the finite-element model. (B) Close up of segmentation between artery and periarterial space as 2D coronal slices (perpendicular to cross-section shown in (A)) along the left branch of the Circle of Willis, where lines depict the edges of tetrahedral mesh vertices. The width of the periarterial space ranges from three to nine vertices. Because the periarterial space is defined primarily by concentration, sometimes a buffer one or two vertices thick resides between the artery and the periarterial space that is indicative of boundaries between regions where one or more materials may reside in the same voxel and influence the measured signal (see Sources of Error). The artery, which holds no contrast agent, exhibits no post-contrast signal change, while the periarterial space, which has high contrast concentration, exhibits large post-contrast signal change. The intermediate region is a combination of both, which produces a signal in between thus excluding the voxel from the definitions of either the arterial or periarterial region. (C) 3D 'crystal brain' image of the surface (light grey) and branching (peach) periarterial subdomains. Translucent white shows the surface of the brain. (D) 3D 'crystal brain' image of arterial (red) and ventricular (blue) subdomains (lateral ventricle (LV), Third Ventricle (3V), Fourth Ventricle (4V), Circle of Willis (CoW).) 


\section{Details of Brain Tissue Subdomain}

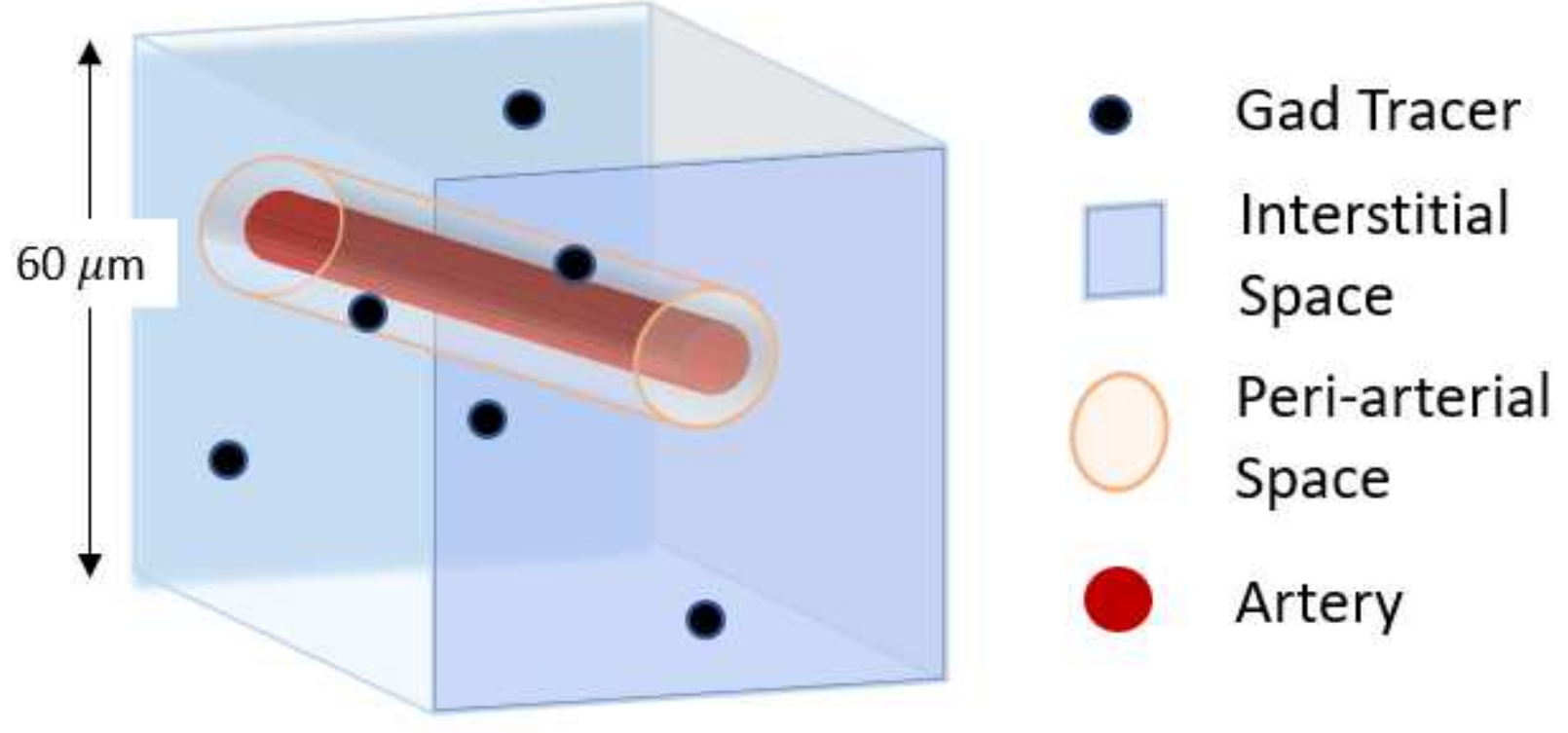

Figure 5

Simplified illustration of a DCE-MRI voxel, which measures $60 \mu \mathrm{m}$ on a side, in the brain tissue subdomain (light blue in Figure 3A). Each voxel in the brain tissue subdomain contains interstitial space and PVSs too small to resolve by MRI. Therefore, the transport parameter determined for this subdomain combines transport in the interstitial space and PVSs. 


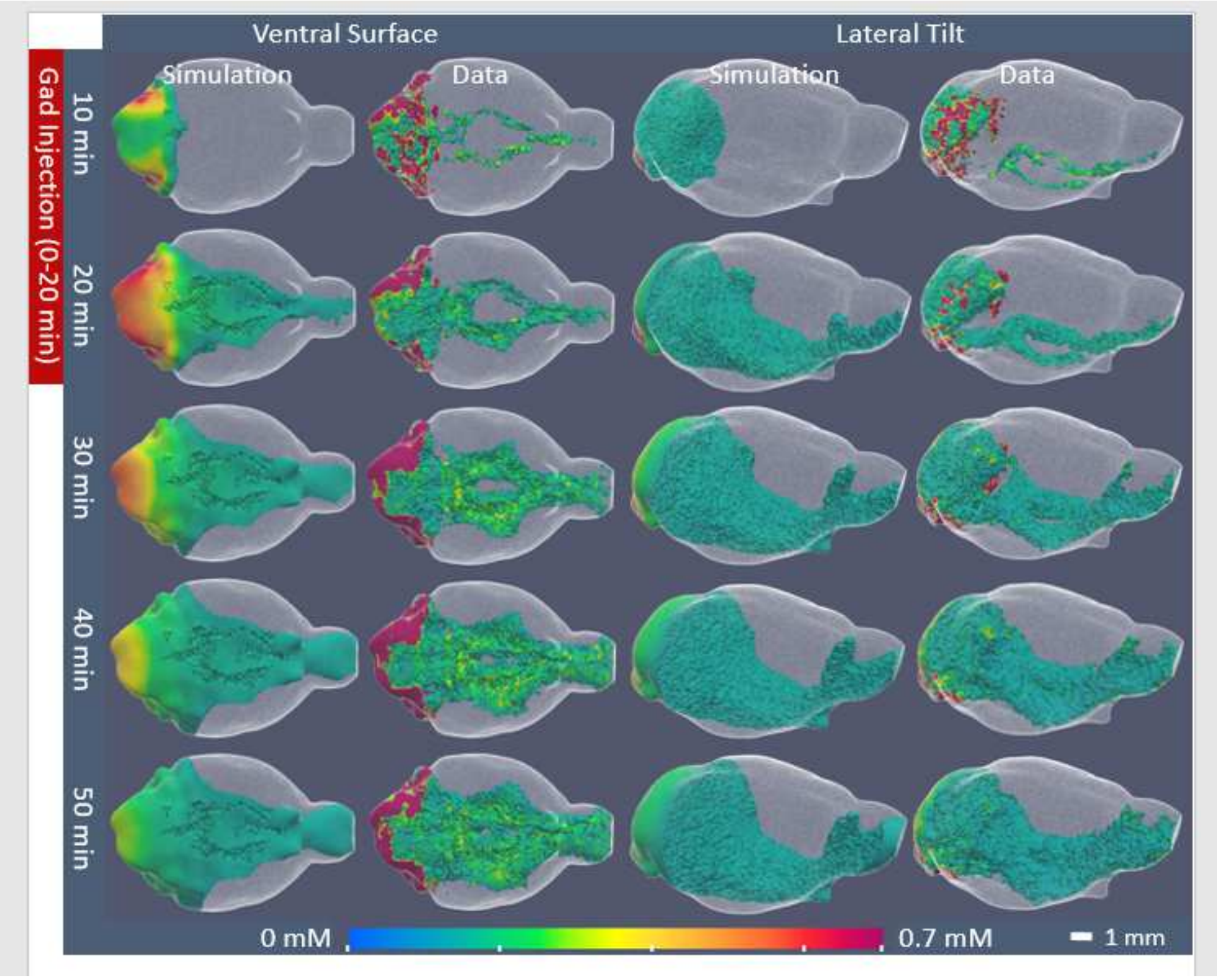

\section{Figure 6}

Comparison of simulation to experimental DCE-MRI concentration data. Images of contrast concentration versus time of simulation results for a representative mouse using segmentation as described above, and optimal transport parameters (brain tissue D_eff $=0.1 \mathrm{~mm} 2 / \mathrm{min}$, PASSurf D_eff $=95 \mathrm{~mm} 2 / \mathrm{min}$, PASBranch D_eff $=60 \mathrm{~mm} 2 / \mathrm{min}$ ) compared to concentration calculated from DCE-MRI signal data. The 3D images show only the elements with concentration greater than $0.1 \mathrm{mM}$ and are colored by concentration according to the color bar at the figure bottom. Images are shown for the ventral surface and from a lateral view with a slight downward tilt to display details around the Circle of Willis on the ventral surface and the branching arteries. In the data images, contrast is observed moving outwards from the injection site near the cisterna magna, rapidly along the ventral surface of the brain following the communicating arteries of the Circle of Willis. Contrast then moves into the brain along major branching arteries, and from these preferential routes, penetrates the wider brain tissue. Contrast present in the ventricular system has been removed from the images for direct comparison to the simulation, 
which modelled only glymphatic transport (not ventricular transport). In the simulations, contrast movement follows the same progression, although it does not move as rapidly towards the anterior brain at early time points and remains higher in posterior regions than in the experimental data.

\section{Supplementary Files}

This is a list of supplementary files associated with this preprint. Click to download.

- FBCNSFigS1.pptx

- FBCNSFigS3.pptx

- FBCNSSupplemental.docx 